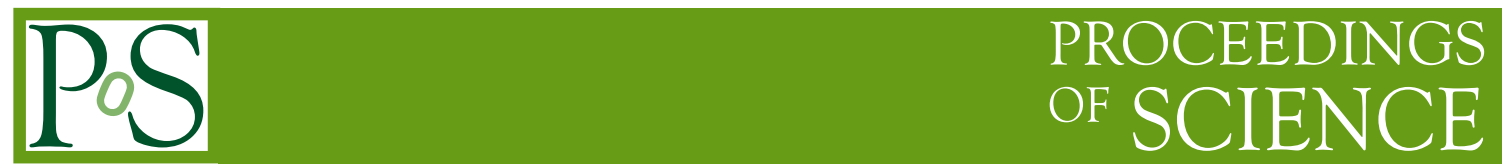

\title{
Analysis of differences on pseudorapidity multiplicities at LHC and UA1/UA5 experiments
}

\author{
Dmitriev Aleksandr \\ Novgorod state university \\ AlexadndrVDmitriev@gmail.com
}

Inconsistencies in rapidity and transverse spectra in ATLAS/ALICE and UA1/UA5 experiments are discussed. It is shown, that rapidity UA1 spectra have additional systematics errors, and $p_{t}$ spectra are more generic. So, actual differences between $p p$ and $p \bar{p}$ interactions in $p_{t}$ distribustions are confirmed.

XXI International Baldin Seminar on High Energy Physics Problems,

September 10-15, 2012

JINR, Dubna, Russia 


\section{Introduction}

Background of this work is an ATLAS CMS and ALICE experimental observation that UA1 $p_{t}$ spectrum is significantly higher (by $\sim 1.15$ ) than LHC one. This effect can be explained [1] by actual difference at $p p$ and $p \bar{p}$ inclusive spectra, but experimenalists explain this observation by UA1 specific procdeure of selecting NSD events by two-arm trigger. Analysis of two-arm UA1 trigger and comparing one with ATLAS, ALICE and CMS triggers is not a subject of this work.

Measured rapidity spectra are, in contrast, equal or slightly lower for UA1/UA5 than ATLAS/ALICE data. In this work we try to explain this inconsistensy between rapidity and $p_{t}$ spectra.

\section{Analisys of pseudirapidity spectra}

ATLAS and UA1 experiments reports pseudorapidity multiplicity spectra at different transverse momentum ranges, whole range $p_{t}>250 \mathrm{Gev}$ for UA1 and $p_{t}>500 \mathrm{Mev}$ [2] for ATLAS. So, to compare these data, one should to estimate $\frac{d N}{d \eta}\left(p_{t}>500 \mathrm{Mev}\right)$ from generic UA1 data and compare with ATLAS data. UA1 gave parametrisation [3] of $p_{t}$ distribution in form

$$
E \frac{d^{3} \sigma}{d p^{3}}=A\left(1+p_{t} / p_{t 0}\right)^{-n}
$$

with $A=382, p_{t 0}=1.56, n=9.96$ at $\sqrt{s}=900 \mathrm{GeV}$.

From this parametrisation, we get for observables rapidity spectra:

$$
\begin{aligned}
& \frac{d N}{d \eta}\left(p_{t}>500 \mathrm{Mev}\right)=A \times \int_{500 \mathrm{Mev}}^{\infty} p_{t}\left(1+p_{t} / p_{t 0}\right)^{-n} d p_{t} \\
& \frac{d N}{d \eta}\left(p_{t}>250 \mathrm{Mev}\right)=A \times \int_{250 \mathrm{Mev}}^{\infty} p_{t}\left(1+p_{t} / p_{t 0}\right)^{-n} d p_{t}
\end{aligned}
$$
$3.48 m b$ :

So, we can get estimation for ATLAS data $\frac{d N}{d \eta}\left(p_{t}>500 \mathrm{Mev}\right)$ from UA1 data $\frac{d N}{d \eta}\left(p_{t}>250 \mathrm{Mev}\right)=$

$$
\begin{array}{r}
\frac{d N}{d \eta}\left(p_{t}>500 \mathrm{Mev}\right)=\frac{\int_{500 \mathrm{Mev}}^{\infty} p_{t}\left(1+p_{t} / p_{t 0}\right)^{-n} d p_{t}}{\int_{0 \mathrm{Mev}}^{\infty} p_{t}\left(1+p_{t} / p_{t 0}\right)^{-n} d p_{t}}= \\
=3.48 \mathrm{mb} * 0.32=1.11 \mathrm{mb}
\end{array}
$$

The ATLAS value $\sim 1.35 \mathrm{mb}$ is higher than this estimation.

ALICE [4] and CMS get the same or slightly higher multiplicity, than UA1 and UA5 experiments, see Fig.1. This values of $\frac{d N}{d \eta}$ is in clear contrast with analysis of $p_{t}$ spectra, there UA1 data is $15 \%$ higher than ATLAS, CMS an ALICE data. So, the question is, how to remove this inconsistency and what kind of data we must prefer for analysis.

ALICE data is accurate enought down to $100 \mathrm{Mev}$. Fraction of particles in umeasured area is above 5\%. In UA1 and UA5 data obtained only down to $250 \mathrm{Mev}$. Fraction of particles in umeasured area is above $35 \%$. So, the ambiguity of continuation to low transverce momenta may be sufficient and give additional systematic uncertainty to rapidity spectrum.

Equality of $p p$ and $p \bar{p}$ inclusive cross sections is commonly used, but this work is based on assumption about significant difference of $p p$ and $p \bar{p}$ inclusive cross sections [1], and, so, we 


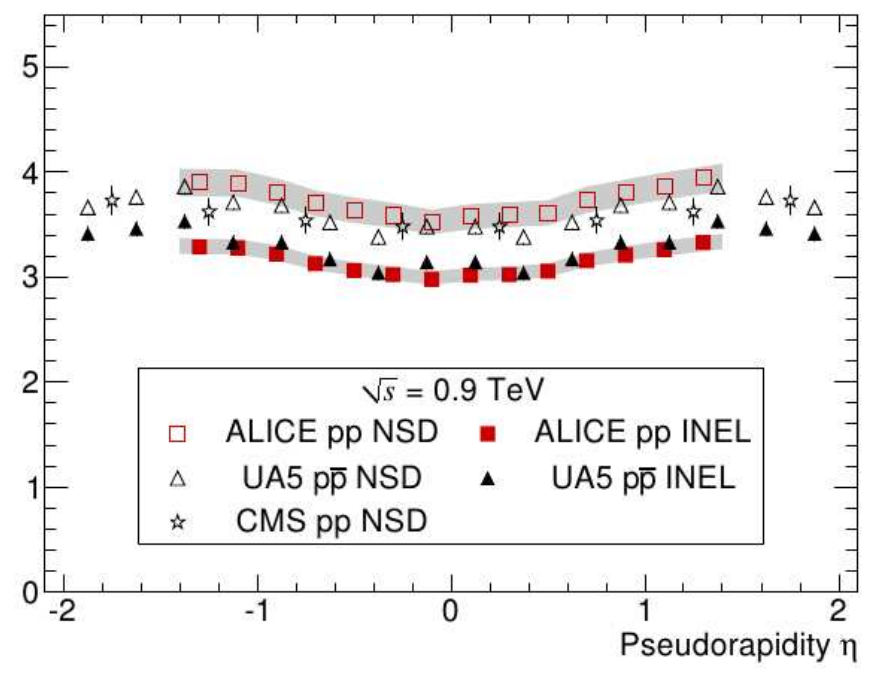

Figure 1: Pseudorapidity measured spectra from UA5, ALICE and CMS experiments.

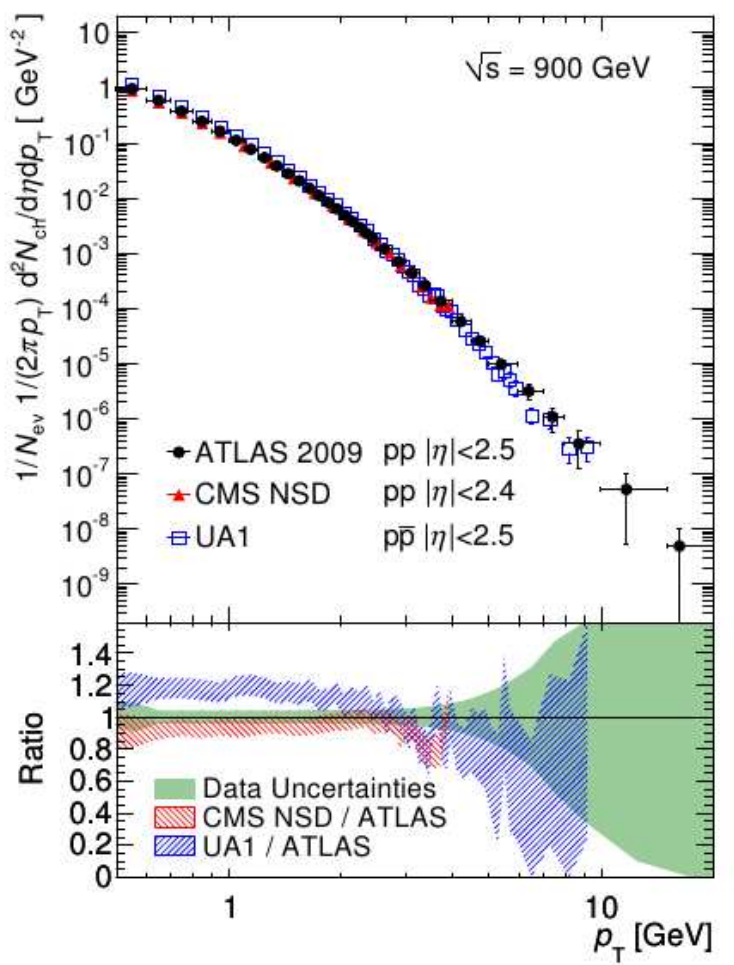

Figure 2: Transverse momentum measured spectra from UA1, ATLAS and CMS experiments. 


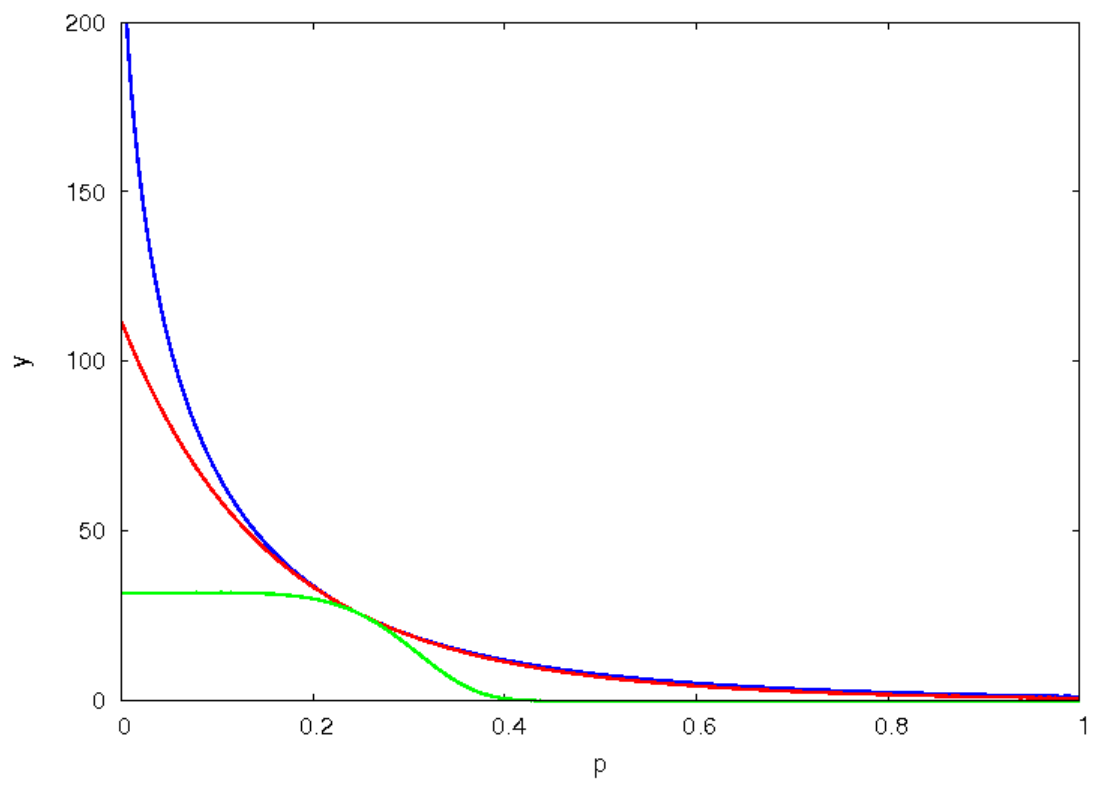

Figure 3: Possible continuation of $\frac{1}{p_{t}} \frac{d N}{d p_{t} d \eta}$ to low $p_{t}$ values. Blue line is higher hypotesys $\sim 1 / p^{0.6}$, red line is original UA1 continuation and green line is saturation (constant) variant.

can not use ALICE (or other LHC experiments) data for determination of continuation UA1 $p \bar{p}$ spectrum to low $p_{t}$.

In UA1 original paper exponential continuation to low $p_{t}$ was developed:

$$
\begin{gathered}
E \frac{d^{3} \sigma}{d p^{3}}=B e^{-b m_{t}} \text { for } p_{t}<p_{t}^{*} \\
m_{t}=\sqrt{m_{\pi}^{2}+p_{t}^{2}} \\
E \frac{d^{3} \sigma}{d p^{3}}=A\left(1+p_{t} / p_{t 0}\right)^{-n} \text { for } p_{t}>p_{t}^{*}
\end{gathered}
$$

This exponential modification does not influence significantly to measured rapidity spectra and averaged $p_{t}$. Let's estimate, how other modifications of low transverse momentum spectra influence on measured values.

Highest hypotesys is motivated by generator simulations with $\sim 1 / p^{0.6}$ peak around $p_{t}=0$, lowest physically motivated hypotesys on behavior of spectrum is that spectrum $\frac{1}{p_{t}} \frac{d N}{d p_{t} d \eta}$ saturates at low $p t$. Both curves and Tsallis parametrisation are shown at Fig.3. Actually, difference is no so high, because of jacobian factor $p_{t}$, and estimations for $\frac{d N}{d p_{t} d \eta}$ shown at Fig.4.

After integration one gets

$$
\begin{aligned}
\text { high hypotesys } \frac{d N}{d \eta}=3.94<p_{t}> & =0.436 \mathrm{GeV} \\
\text { actual data } \frac{d N}{d \eta}=3.8<p_{t}> & =0.448 \mathrm{GeV} \\
\text { low hypotesys } \frac{d N}{d \eta}=3.39<p_{t}> & =0.49 \mathrm{GeV}
\end{aligned}
$$




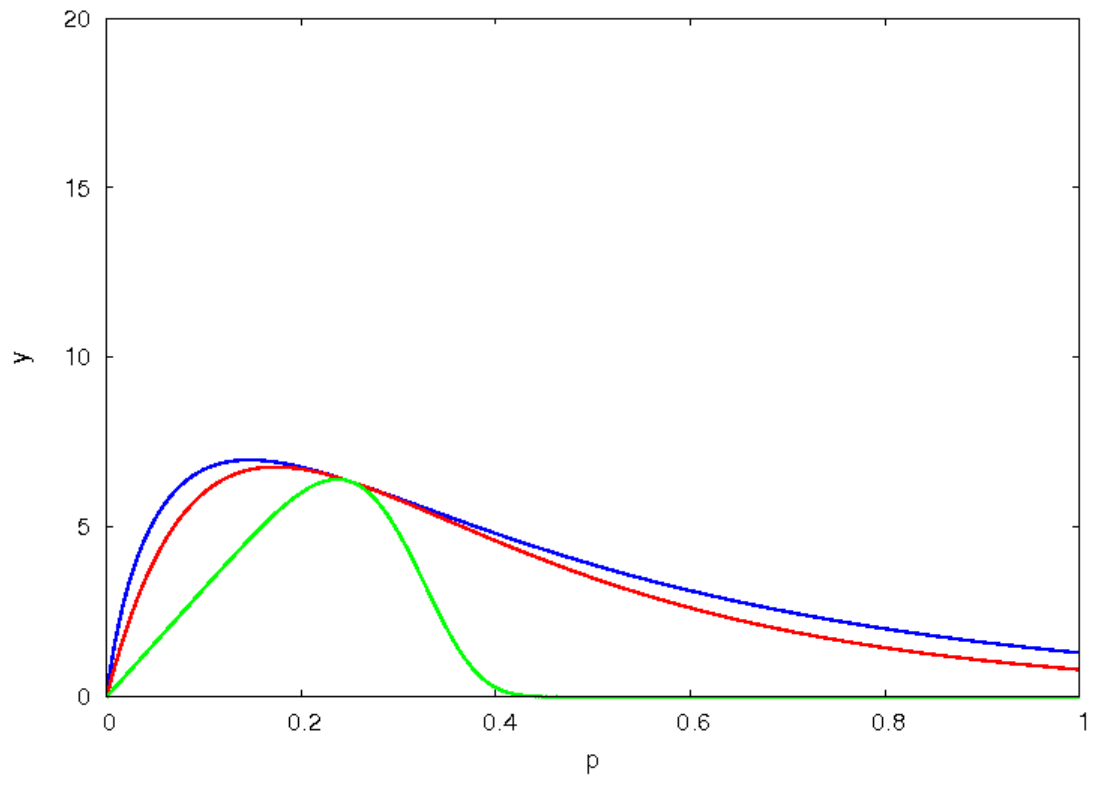

Figure 4: Possible continuation of $p_{t} \frac{1}{p_{t}} \frac{d N}{d p_{t} d \eta}$ to low $p_{t}$ values. Blue line is higher hypotesys $\sim 1 / p^{0.6}$, red line is original UA1 continuation and green line is saturation (constant) variant.

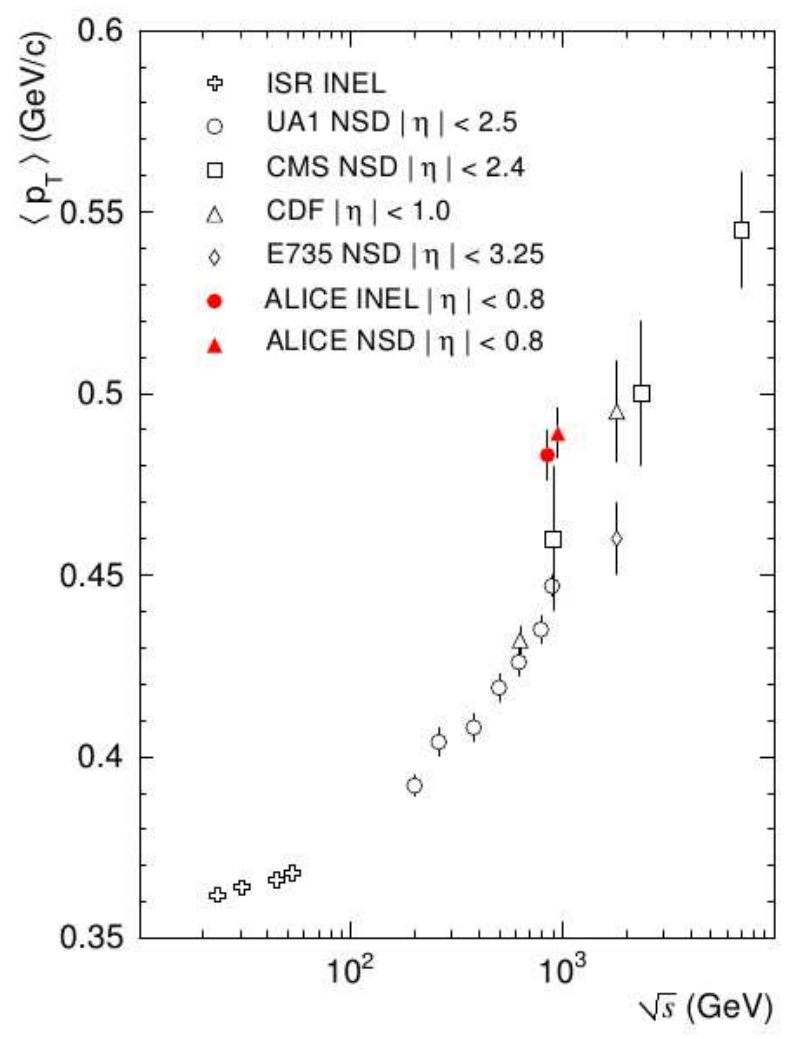

Figure 5: Average transverse momentum for $p p$ and $p \bar{p}$ experiments. ALICE $p p$ average momentum is significantly higher than CMS and UA1 $p \bar{p}$ values. 
So, additional systematic uncertanity on UA1 rapidity spectrum $\frac{d N}{d \eta}$ is estimated abot $15 \%$, which makes rapidity spectra data compatable with transverse momenta spectra data.

Average transverse momentum value is also changed by low- $p_{t}$ spectrum variation, and it can explaines difference between ALICE and CMS/UA1 data, see Fig.5

\section{Conclusion}

Equality of rapidity spectra for $p p$ and $p \bar{p}$ is not surely stated, while transverse momentum spectrum is more generic and clearly shows difference between $p p$ and $p \bar{p}$ inclusive spectra.

\section{Acknowledgments}

The author is grateful for Prof. V.A.Abramovsky and N.V.Prikhod'ko for useful discussion and comments. The work is supported by RFBR grant 11-02-01395-a.

\section{References}

[1] V. A. Abramovsky and N. V. Radchenko, Possible difference between multiplicity distributions and inclusive spectra of secondary hadrons in proton-proton and proton-antiproton collisions at energy s** $(1 / 2)=900-G e V, "$ arXiv:0912.1041 [hep-ph].

[2] G. Aad et al. [ATLAS Collaboration], Phys. Lett. B 688 (2010) 21 [arXiv:1003.3124 [hep-ex]].

[3] C. Albajar et al. [UA1 Collaboration], Nucl. Phys. B 335 (1990) 261.

[4] K. Aamodt et al. [ALICE Collaboration], Eur. Phys. J. C 68 (2010) 89 [arXiv:1004.3034 [hep-ex]]. 